\title{
Representations for the Drazin inverses of block matrices
}

Li Guo ${ }^{1,2}$, Jie Wang ${ }^{2}$, Wenming $\mathrm{Du}^{2}$

${ }^{1}$ Department of Mathematics, Southeast University, Nanjing, china

${ }^{2}$ College of Mathematics, Beihua University, Jilin, china

\begin{abstract}
In this paper, we investigate representations of the Drazin inverse of a $2 \times 2$ block matrix. The Drazin inverse of a matrix is very important in various applied mathematical fields like machinery and automation, singular differential equations. We give a explicit representation of the Drazin inverse of a block matrix.
\end{abstract}

Keywords: Block matrix; Drazin inverse; Index

\section{Introduction}

Let $C^{n \times n}$ denote the set of $n \times n$ complex matrices. The smallest nonnegative integer $k$ such that $\operatorname{rank}\left(A^{k+1}\right)=\operatorname{rank}\left(A^{k}\right)$, denoted by $\operatorname{ind}(A)$, is called the index of $A$. The Drazin inverse is the unique matrix $A^{d} \in C^{n \times n}$ satisfying

$$
A A^{d}=A^{d} A, A^{d} A A^{d}=A^{d}, A^{k+1} A^{d}=A^{k},
$$

where $k=\operatorname{ind}(A)$. We denote by $A^{\pi}=I-A A^{d}$. For $A \in C^{n \times n}$ we define $A^{0}=I_{n}$, the identity of order $n$, even if $A=0$.

The Drazin inverse of a matrix is very important in various applied mathematical fields like machinery and automation, singular differential equations, singular difference equations, Markov chains, iterative methods and so on $[1,2]$. We introduce briefly an application of the Drazin inverse of a block matrix[2].
In this paper, representations for the Drazin inverse of $2 \times 2$ block matrix $M$, under the following conditions that $B D=0, \quad D^{\pi} C B=0, \quad D^{\pi} C A B=0, \quad D^{\pi} C A^{2}=0$ and so several results are generalized.

Lemma 1.1 [3] Let $A \in C^{m \times n}$ and $B \in C^{n \times m}$, then $(A B)^{d}=A\left[(B A)^{2}\right]^{d} B$.

Lemma 1.2 Let $A \in C^{m \times m}$, then $\left(A^{2}\right)^{d}=\left(A^{2}\right)^{2}$.

Lemma 1.3 (see [5],) Let $M=\left(\begin{array}{ll}A & B \\ C & D\end{array}\right)$ be a $2 \times 2$ block complex matrix, where $A \in C^{m \times m}$ and $D \in C^{n \times n}$ with $\operatorname{ind}(A)=r$, ind $(D)=s$, if $B C=0, B D=0$ then and $k=0,1$,

$$
M^{d}=\left(\begin{array}{cc}
A^{d} & \left(A^{d}\right)^{2} B \\
\sum_{0} & D^{d}+\sum_{1} B
\end{array}\right),
$$

$$
\sum_{k}=\left(D^{d}\right)^{2} \sum_{i=0}^{r-1}\left(D^{d}\right)^{i+k} C A^{i} A^{\pi}+D^{\pi} \sum_{i=0}^{s-1} D^{i} C\left(A^{d}\right)^{i+k}\left(A^{d}\right)^{2}-\sum_{i=0}^{k}\left(D^{d}\right)^{i+1} C\left(A^{d}\right)^{k-i+1} .
$$

with $\operatorname{ind}(M) \leq \operatorname{ind}(A)+\operatorname{ind}(D)+1$.

$$
\text { ind }(P)=r \text {, ind }(Q)=s \text {, if }
$$

$P Q P=0, P Q^{2}=0$ then

Lemma 1.4 (see [5],) Let $P, Q \in C^{n \times n}$, where 


$$
\begin{aligned}
(P+Q)^{d} & =Q^{\pi} \sum_{i=0}^{s-1} Q^{i}\left(P^{D}\right)^{i+1}+\sum_{i=0}^{r-1}\left(Q^{D}\right)^{i+1}\left(P^{i}\right) P^{\pi}+Q^{\pi} \sum_{i=0}^{s-1} Q^{i}\left(P^{D}\right)^{i+2} Q \\
& +\sum_{i=0}^{r-2}\left(Q^{D}\right)^{i+3}\left(P^{i+1}\right) P^{\pi} Q Q^{D} P^{D} Q-\left(Q^{D}\right) P P^{D} Q .
\end{aligned}
$$

\section{Main Results}

Theorem 2.1 Let $M=\left(\begin{array}{ll}A & B \\ C & D\end{array}\right)$ be a $2 \times 2$ block complex matrix, where $A \in C^{m \times m}$ and $D \in C^{n \times n}$ with $\operatorname{ind}(A)=r$, ind $(D)=s$, if

then $B D=0, \quad D^{\pi} C B=0, \quad D^{\pi} C A B=0, \quad D^{\pi} C A^{2}=0$,

$$
M^{D}=\left(\begin{array}{cc}
A^{D}+\left(A^{D}\right)^{3} B C+\left(A^{D}\right)^{4} B C A & \left(A^{D}\right)^{2} B+\left(A^{D}\right)^{4} \mathrm{BCB} \\
\psi_{0}+\psi_{0}\left(A^{D}\right)^{2} B C+D^{D} \psi_{1} B C+\Delta D D^{D} C & D^{D}+\psi_{1} B+\Delta B D^{\pi}
\end{array}\right),
$$

where

$$
\begin{gathered}
\Delta=\psi_{0}\left(A^{D}\right)^{3} B C+D^{D} \psi_{0}\left(A^{D}\right)^{2} B C+\psi_{0} D^{D} \psi_{1} B C, \\
\psi_{k}=\sum_{i=0}^{r-1}\left(D^{D}\right)^{i+k+2} C A^{i} A^{\pi}-\sum_{i=0}^{k}\left(D^{D}\right)^{i+1} C\left(A^{D}\right)^{k-i+1}, k=0,1 .
\end{gathered}
$$

Proof. Let $M=P+Q$, where

$$
P=\left(\begin{array}{cc}
0 & B D D^{D} \\
D^{\pi} C & 0
\end{array}\right), Q=\left(\begin{array}{cc}
A & B D^{\pi} \\
D D^{D} C & D
\end{array}\right) .
$$

By $B D=0, D^{\pi} C B=0$, then

$$
\begin{aligned}
P Q P & =\left(\begin{array}{cc}
0 & B D D^{\pi} \\
D^{\pi} C & 0
\end{array}\right)\left(\begin{array}{cc}
A & B D^{\pi} \\
D D^{D} C & D
\end{array}\right)\left(\begin{array}{cc}
0 & B D D^{D} \\
D^{\pi} C & 0
\end{array}\right) \\
& =\left(\begin{array}{cc}
B D D^{D} \mathrm{C} & B D^{2} D^{D} \\
D^{\pi} C A & D^{\pi} C B D^{\pi}
\end{array}\right)\left(\begin{array}{cc}
0 & B D D^{D} \\
D^{\pi} C & 0
\end{array}\right) \\
& =0 .
\end{aligned}
$$

Since $D^{\pi} C B=0, D^{\pi} C A B=0, D^{\pi} C A^{2}=0$, we have

$$
\begin{aligned}
P Q^{2} & =\left(\begin{array}{cc}
0 & B D D^{D} \\
D^{\pi} C & 0
\end{array}\right)\left(\begin{array}{cc}
A & B D^{\pi} \\
D D^{D} C & D
\end{array}\right)\left(\begin{array}{cc}
A & B D^{\pi} \\
D^{D} D C & D
\end{array}\right) \\
& =\left(\begin{array}{cc}
B D D^{D} \mathrm{C} & B D^{2} D^{D} \\
D^{\pi} C A & D^{\pi} C B D^{\pi}
\end{array}\right)\left(\begin{array}{cc}
A & B D^{\pi} \\
D D^{D} C & D
\end{array}\right) \\
& =\left(\begin{array}{cc}
B D D^{D} C A+B D^{2} D^{D} C & B D D^{D} C B D^{D}+B D^{3} D^{D} \\
D^{\pi} C A^{2} & D^{\pi} C A B D^{\pi}+D^{\pi} C B D^{\pi} \mathrm{D}
\end{array}\right) \\
& =0 .
\end{aligned}
$$

By $P^{2}=0$, we get that $P^{D}=0, P^{\pi}=I$. Noting that $B D=0$, by Lemma 1.3 , we have

$$
Q^{D}=\left(\begin{array}{cc}
A^{D} & \left(A^{D}\right)^{2} B \\
\psi_{0} & D^{D}+\psi_{1} B
\end{array}\right)
$$

where 


$$
\psi_{k}=\sum_{i=0}^{r-1}\left(D^{D}\right)^{i+k+2} C A^{i} A^{\pi}-\sum_{i=0}^{k}\left(D^{D}\right)^{i+1} C\left(A^{D}\right)^{k-i+1}, \quad k=0,1 .
$$

Since $P Q P=0, P Q^{2}=0$, By Lemma 1.4, we

get

$$
\begin{gathered}
(P+Q)^{D}=Q^{\pi} \sum_{n=0}^{k-1} Q^{i}\left(P^{D}\right)^{i+1}+\sum_{i=0}^{m-1}\left(Q^{D}\right)^{i+1} P^{i} P^{\pi}+Q^{\pi} \sum_{i=0}^{k-1} Q^{i}\left(P^{D}\right)^{i+2} Q \\
+\sum_{i=0}^{m-2}\left(Q^{D}\right)^{i+3} P^{i+1} P^{\pi} Q-Q^{D} P^{D} Q-\left(Q^{D}\right)^{2} P P^{D} Q,
\end{gathered}
$$

where

$$
k=\operatorname{ind}(Q) \leq r+s+1, \quad m=\operatorname{ind}(P)=2,
$$

By $P^{D}=0, P^{\pi}=I$, then

$$
\begin{aligned}
(P+Q)^{D} & =\sum_{i=0}^{m-1}\left(Q^{D}\right)^{i+1} P^{i}+Q^{\pi} \sum_{i=0}^{m-2}\left(Q^{D}\right)^{i+3} P^{i+1} Q \\
& =Q^{D}+\left(Q^{D}\right)^{2} P+\left(Q^{D}\right)^{3} P Q \\
\left(Q^{D}\right)^{2} P & =\left(\begin{array}{cc}
A^{D} & \left(A^{D}\right)^{2} B \\
\psi_{0} & D^{D}+\psi_{1} \mathrm{~B}
\end{array}\right)\left(\begin{array}{cc}
\left(A^{D}\right)^{2} B D^{\pi} C & 0 \\
\psi_{1} B D^{\pi} C & 0
\end{array}\right) \\
& =\left(\begin{array}{ccc}
\left(A^{D}\right)^{3} B C+\left(A^{D}\right)^{2} \psi_{1} B C & 0 \\
\psi_{0}\left(A^{D}\right)^{2} B C+D^{D} \psi_{1} B C+\psi_{1} B \psi_{1} B C & 0
\end{array}\right) .
\end{aligned}
$$

By $B D=0, B D^{D}=0, B \psi_{1}=0$, so we have

$$
\left(Q^{D}\right)^{2} P=\left(\begin{array}{cc}
\left(A^{D}\right)^{3} B C & 0 \\
\psi_{0}\left(A^{D}\right)^{2} B C+D^{D} \psi_{1} B C & 0
\end{array}\right) .
$$

By $B \psi_{0}=0, B \psi_{1}=0, B D^{D}=0$, we get

$$
\begin{aligned}
\left(Q^{D}\right)^{3} P & =\left(\begin{array}{cc}
A^{D} & \left(A^{D}\right)^{2} B \\
\psi_{0} & D^{D}+\psi_{1} \mathrm{~B}
\end{array}\right)\left(\begin{array}{cc}
\left(A^{D}\right)^{3} B C & 0 \\
\psi_{0}\left(A^{D}\right)^{2} B C+D^{D} \psi_{1} B C & 0
\end{array}\right) \\
& =\left(\begin{array}{cc}
\left(A^{D}\right)^{4} B C & 0 \\
\psi_{0}\left(A^{D}\right)^{3} B C+D^{D} \psi_{0}\left(A^{D}\right)^{2} B C+\psi_{0} D^{D} \psi_{1} B C & 0
\end{array}\right),
\end{aligned}
$$

and

$$
\begin{aligned}
\left(Q^{D}\right)^{3} P Q & =\left(\begin{array}{cc}
\left(A^{D}\right)^{4} B C & 0 \\
\psi_{0}\left(A^{D}\right)^{3} B C+D^{D} \psi_{0}\left(A^{D}\right)^{2} B C+\psi_{0} D^{D} \psi_{1} B C & 0
\end{array}\right)\left(\begin{array}{cc}
A & B D^{\pi} \\
D D^{D} C & \mathrm{D}
\end{array}\right) \\
& =\left(\begin{array}{cc}
\left(A^{D}\right)^{4} B C A & \left(A^{D}\right)^{4} B C B \\
\Delta D D^{D} \mathrm{C} & \Delta \mathrm{BD}^{\pi}
\end{array}\right) .
\end{aligned}
$$

where

$$
\Delta=\psi_{0}\left(A^{D}\right)^{3} B C+D^{D} \psi_{0}\left(A^{D}\right)^{2} B C+\psi_{0} D^{D} \psi_{1} B C .
$$

So we have 


$$
\begin{aligned}
(P+Q)^{D} & =Q^{D}+\left(Q^{D}\right)^{2} P+\left(Q^{D}\right)^{3} P Q \\
& =\left(\begin{array}{cr}
A^{D}+\left(A^{D}\right)^{3} B C+\left(A^{D}\right)^{4} B C A & \left(A^{D}\right)^{2} B+\left(A^{D}\right)^{4} B C B \\
\psi_{0}+\psi_{0}\left(A^{D}\right)^{2} B C+D^{D} \psi_{1} B C+\Delta D D^{D} C & D^{D}+\psi_{1} B+\Delta B D^{\pi}
\end{array}\right) .
\end{aligned}
$$

This result generalizes the result under the condition $B D=0, D^{\pi} C B=0, D^{\pi} C A=0$ in [4]

\section{References}

[1] Campbell S L, Meyer C D, Jr. Generalized Inverses of Linear Transformations. London: Pitman Press, 1979.

[2] Campbell S L. The Drazin inverse and systems of second order linear differential equations. Linear Multili -near Algebra, Vol. 14 (1983), 195-198.
[3] D. S. Djordjevic, Y. Wei,, Additive results for the generalized Drazin inverse, J. Aust. Math. Soc. Vol. 73 (2002), 115 -126,

[4] Dopazo E, Matrinez-Serrano M F. Further results on the representation of the Drazin inverse of a $2 \times 2$ block matrix. Linear Algebra Appl, Vol. 43 (2010), 1896-1904.

[5] Li Guo, Xiankun Du. Representations for the Drazin inverses of $2 \times 2$ block matrices. Applied Mathematics and Computation, Vol. 217 (2010), 2833-2842. 